\title{
Isolated Adrenocorticotropic Hormone Deficiency Caused by Nivolumab in a Patient with Metastatic Lung Cancer
}

\author{
Kazuhiko Takaya, Miwa Sonoda, Ayako Fuchigami and Toru Hiyoshi
}

\begin{abstract}
:
Nivolumab has promising efficacy for treating various advanced malignant tumors, although it has been reported to induce a wide range of autoimmune adverse effects. We herein report the case of a patient with metastatic lung adenocarcinoma who developed adrenal insufficiency after 12 cycles of nivolumab treatment. Endocrine test results supported a diagnosis of isolated adrenocorticotropic hormone deficiency due to hypophysitis, and replacement therapy using hydrocortisone has been successful. Although hypophysitis is a rare immune-related adverse event that is associated with nivolumab therapy, clinical awareness is essential, as this condition can be life-threatening and requires prompt treatment.
\end{abstract}

Key words: hypophysitis, adrenal insufficiency, nivolumab, lung cancer

(Intern Med 56: 2463-2469, 2017)

(DOI: 10.2169/internalmedicine.8548-16)

\section{Introduction}

Immune checkpoints help maintain peripheral tolerance to self-molecules and include co-inhibitory molecules, such as cytotoxic T-lymphocyte-associated protein 4 (CTLA-4), programmed cell death 1 (PD-1), lymphocyte-activation gene 3 , T-cell immunoglobulin mucin-3, and co-stimulatory molecules (e.g. glucocorticoid-induced tumor necrosis factor receptor and OX40/CD134) (1). Tumor cells can avoid immune destruction through many mechanisms, including passing through these checkpoints, and monoclonal antibodies that block co-inhibitory immune checkpoint molecules can help increase the T-cell specific immune response that facilitates the immune destruction of tumor cells. Recently developed immunotherapeutic agents that target immune checkpoints can enhance the innate immune response and have promising efficacies for treating an expanding list of metastatic tumors $(2,3)$. Ipilimumab is a monoclonal IgG1 antibody that blocks the function of CTLA-4 and, in 2011, was the first immunotherapeutic agent to be approved in the US for treating advanced malignant melanoma. There are currently 142 open clinical trials for ipilimumab according to ClinicalTrials.gov (4).

The blockade of PD-1 or its ligands (PD-L1 and PD-L2) is a potential alternative treatment for advanced malignant tumors. PD-1 is an inhibitory receptor expressed on the surface of activated T- and B-lymphocytes and monocytes. The binding of PD-L1 and PD-L2 to PD-1 on immune cells inhibits the immune response of these cells. PD-L1 and PD-L 2 may be present on some malignant cells, possibly as a mechanism to escape immune surveillance (5). Nivolumab is a monoclonal IgG4 antibody that binds PD-1 and helps destroy cancer cells through a T-cell activation mechanism $(2,3,6)$. Nivolumab has been approved for treating various malignant diseases (e.g. malignant melanoma, nonsmall cell lung cancer, and renal cell carcinoma) in many countries (7), and ClinicalTrials.gov lists 231 open clinical trials (4).

In addition to their antitumor effects, immune checkpoint inhibitors can induce a wide range of autoimmune adverse effects, namely immune-related adverse events (IRAEs), such as colitis, dermatitis, hepatitis, pancreatitis, and other rare events, e.g. nephritis, polymyositis, uveitis, toxic epidermal necrolysis, drug reaction with eosinophilia and systemic symptoms (DRESS) syndrome, hemophilia A, and TolosaHunt syndrome (8). These agents can also trigger endocrine IRAEs, which include hypophysitis, thyroiditis, hypothyroidism, hyperthyroidism, Graves' ophthalmopathy, primary adrenal insufficiency, and type 1 diabetes (7). Among these 
IRAEs, a relatively high incidence of hypophysitis is associated with ipilimumab therapy, and hypophysitis is extremely rare in patients treated using other immune check point inhibitors (ICIs) (5).

In this report, we describe a patient with metastatic lung cancer who developed severe adrenal insufficiency due to isolated adrenocorticotropic hormone deficiency after 12 cycles of nivolumab therapy. We also discuss the characteristics of nivolumab-induced hypophysitis, compared to ipilimumab-induced hypophysitis.

\section{Case Report}

A 75-year-old Japanese man had previously undergone multiple treatments for metastatic lung adenocarcinoma (stage IV, cT2aN0M1b). His original lesion had been discovered 2.5 years previously, in the apical segment of the right lung with metastasis to the right cingulate gyrus of the brain. He had received six cycles of carboplatin-pemetrexed chemotherapy for the original lesion and undergone stereotactic irradiation of the original and metastatic lesions. His disease progressed despite these treatments, although the irradiation significantly reduced the size of the metastatic brain lesion. He subsequently developed additional metastases in his sternum, left pubis, mediastinal lymph nodes, and left adrenal gland and started to complain of dyspnea on effort due to pleural effusion. He was treated using nivolumab monotherapy ( $3 \mathrm{mg} / \mathrm{kg}$ every 2 weeks), and the possibility of nivolumab-induced endocrine abnormalities was evaluated using blood tests to monitor his levels of adrenocorticotropic hormone (ACTH), cortisol, thyroid-stimulating hormone (TSH), and thyroid hormones. His disease stabilized, with no remarkable disease development during a five-month period of lung computed tomography (CT) monitoring. His plasma ACTH and serum cortisol levels also remained within the normal ranges during that period.

At two weeks after the twelfth cycle of nivolumab therapy, the patient presented to our clinic with severe progressive fatigue and appetite loss. The symptoms had developed two days after the twelfth cycle of nivolumab therapy, worsening each day, and he was unable to eat at all on the day of his presentation. Adrenal insufficiency was suspected based on his symptoms, and he was hospitalized and underwent emergent endocrinological testing.

At admission, the patient's height was $164.0 \mathrm{~cm}$, his weight was $58.0 \mathrm{~kg}$, his blood pressure was lower than the previous month $(88 / 59 \mathrm{mmHg}$ vs. $123 / 67 \mathrm{mmHg})$, and he had a pulse of 65 beats/min with a regular rhythm. Although he had been afebrile at the presentation $\left(36.7^{\circ} \mathrm{C}\right)$, he subsequently developed a fever of $37.1-38.2^{\circ} \mathrm{C}$ after his hospitalization. He did not report experiencing headache or nausea. The patient's superficial lymph nodes were not palpable, and he did not have an enlarged liver or spleen. His oral cavity and skin were relatively dry, which suggested clinical dehydration. The laboratory test results included normal blood glucose levels, low serum albumin levels, slightly lower se- rum sodium levels, and slightly elevated serum levels of Creactive protein and soluble interleukin-2 receptor (Table 1). Hormone tests revealed markedly low plasma ACTH and serum cortisol levels, with undetected urine levels of free cortisol and slightly elevated serum TSH levels (Table 1). Low plasma renin activity (PRA) associated with a slightly elevated plasma aldosterone concentration (PAC) was also observed (Table 1). We tentatively diagnosed the patient with adrenal insufficiency and immediately started $0.25 \mathrm{mg} /$ day of oral dexamethasone and saline infusion, with additional endocrinological investigations.

Pituitary stimulation testing using a combination of corticotropin-releasing hormone, luteinizing hormonereleasing hormone, and thyrotropin-releasing hormone revealed markedly low ACTH and cortisol responses, with normal responses for luteinizing hormone and folliclestimulating hormone (Fig. 2A). An over-response was observed for prolactin, with a peak value of $>100 \mathrm{ng} / \mathrm{mL}$. A slight over-response was also observed for TSH. A stimulation test using growth hormone-releasing peptide- 2 revealed a normal growth hormone response (Fig. 2B). Adrenal stimulation testing was also performed, and the cortisol response (stimulated using $\mathrm{ACTH}$ ) was delayed and below the normal level (Fig. 2C). Contrast-enhanced magnetic resonance imaging (MRI) of the hypothalamus and the pituitary gland revealed no signs of pituitary swelling, stalk thickness, or a space-occupying lesion (Fig. 1). We also observed a normal high-intensity signal in the posterior lobe on T1weighted images. Chest and abdomen CT revealed the original lesion in lung segment 2, metastatic lesions in the paratracheal area, left pleural effusion, and a left adrenal mass that suggested metastasis, with no significant changes over the previous three months (data not shown).

These results were consistent with a diagnosis of adrenal failure due to isolated-ACTH deficiency, possibly as an IRAE. We therefore administered high-dose intravenous hydrocortisone (150 mg/day) for 1 day, which was subsequently replaced using oral hydrocortisone $(15 \mathrm{mg} /$ day $)$. The patient's symptoms of general fatigue and appetite loss promptly improved within several hours after the high-dose hydrocortisone treatment, and his fever also disappeared immediately. He did not develop thirst or polyuria, even after starting the hydrocortisone, which suggested that he did not have masked diabetes insipidus. His vasopressin levels were measured after the hydrocortisone substitution and were found to be within the appropriate range compared to his serum sodium and plasma osmolality levels (data not shown). An ACTH loading test was performed for five consecutive days after the hydrocortisone replacement. The serum cortisol level in the early morning of the sixth day was $48.1 \mu \mathrm{g} /$ $\mathrm{dL}$, indicating that his adrenal insufficiency had a secondary origin. Serological tests were performed to examine the possible immunological etiology of his ACTH deficiency and to exclude other IRAEs. We observed negative results for antipituitary cell antibody 1 , anti-adrenal cortex antibodies, and other autoantibodies; the only positive result was for anti- 
Table 1. Laboratory Findings.

\begin{tabular}{|c|c|c|c|}
\hline Urinalysis & & $\mathrm{Cl}$ & $100 \mathrm{mEq} / \mathrm{L}$ \\
\hline Protein & $(-)$ & $\mathrm{Ca}$ & $8.0 \mathrm{mg} / \mathrm{dL}$ \\
\hline Occult blood & $(-)$ & IP & $3.2 \mathrm{mg} / \mathrm{dL}$ \\
\hline \multirow[t]{2}{*}{ Gravity } & 1.019 & $\operatorname{IgG}$ & $864 \mathrm{mg} / \mathrm{dL}$ \\
\hline & & IgG4 & $42 \mathrm{mg} / \mathrm{dL}$ \\
\hline Cell blood count & & $\operatorname{IgA}$ & $198 \mathrm{mg} / \mathrm{dL}$ \\
\hline WBC & $4,000 / \mu \mathrm{L}$ & $\operatorname{IgM}$ & $30 \mathrm{mg} / \mathrm{dL}$ \\
\hline Segmented & $66.1 \%$ & $\operatorname{IgE}$ & $167 \mathrm{IU} / \mathrm{mL}$ \\
\hline Stab & $0.0 \%$ & $\operatorname{IgD}$ & $<6 \mathrm{mg} / \mathrm{dL}$ \\
\hline Lymphocyte & $19.5 \%$ & $\mathrm{CH} 50$ & $77 \mathrm{U} / \mathrm{mL}$ \\
\hline Basophil & $0.2 \%$ & $\mathrm{C} 3$ & $133 \mathrm{mg} / \mathrm{dL}$ \\
\hline Eosinophil & $4.7 \%$ & $\mathrm{C} 4$ & $28.4 \mathrm{mg} / \mathrm{dL}$ \\
\hline Monocyte & $9.5 \%$ & $\mathrm{ACE}$ & $8.4 \mathrm{IU} / \mathrm{L}$ \\
\hline $\mathrm{RBC}$ & $393 \times 10^{4} / \mu \mathrm{L}$ & sIL-2R & $607 \mathrm{U} / \mathrm{mL}$ \\
\hline Hemoglobin & $12.2 \mathrm{~g} / \mathrm{dL}$ & & \\
\hline \multirow[t]{2}{*}{ Platelet } & $19.6 \times 10^{4} / \mu \mathrm{L}$ & Endocrinology & \\
\hline & & TSH & $6.88 \mu \mathrm{IU} / \mathrm{mL}$ \\
\hline Blood chemistry & & $\mathrm{FT}_{3}$ & $3.8 \mathrm{pg} / \mathrm{mL}$ \\
\hline Total protein & $5.4 \mathrm{~g} / \mathrm{dL}$ & $\mathrm{FT}_{4}$ & $0.83 \mathrm{ng} / \mathrm{dL}$ \\
\hline Albumin & $2.5 \mathrm{~g} / \mathrm{dL}$ & Free testosterone & $1.3 \mathrm{pg} / \mathrm{mL}$ \\
\hline AST & $22 \mathrm{IU} / \mathrm{L}$ & АCTH & $<1.0 \mathrm{pg} / \mathrm{mL}$ \\
\hline ALT & $11 \mathrm{IU} / \mathrm{L}$ & Cortisol & $0.4 \mu \mathrm{g} / \mathrm{dL}$ \\
\hline ALP & $83 \mathrm{IU} / \mathrm{L}$ & DHEA-S & $<50.0 \mu \mathrm{g} / \mathrm{dL}$ \\
\hline LDH & $283 \mathrm{IU} / \mathrm{L}$ & PRA & $0.2 \mathrm{ng} / \mathrm{mL} / \mathrm{hr}$ \\
\hline CK & $77 \mathrm{U} / \mathrm{L}$ & PAC & $123 \mathrm{pg} / \mathrm{mL}$ \\
\hline Fasting plasma glucose & $103 \mathrm{mg} / \mathrm{dL}$ & IGF-1 & $85 \mathrm{ng} / \mathrm{mL}$ \\
\hline BUN & $12 \mathrm{mg} / \mathrm{dL}$ & iPTH & $41 \mathrm{pg} / \mathrm{mL}$ \\
\hline Creatinine & $0.69 \mathrm{mg} / \mathrm{dL}$ & Urine free cortisol & not detected \\
\hline $\mathrm{Na}$ & $133 \mathrm{mEq} / \mathrm{L}$ & Urine metanephrine & $0.12 \mathrm{mg} /$ day \\
\hline K & $4.5 \mathrm{mEq} / \mathrm{L}$ & Urine normetanephrine & $0.36 \mathrm{mg} / \mathrm{day}$ \\
\hline
\end{tabular}

WBC: white blood cell, RBC: red blood cell, AST: aspartate aminotransferase, ALT: alanine aminotransferase, ALP: alkaline phosphatase, LDH: lactate dehydrogenase, CK: creatine kinase, BUN: blood urea nitrogen, IP: inorganic phosphorus, Ig: immunoglobulin, CH50: complement activities, C3: complement component 3, C4: complement component 4, ACE: angiotensin converting enzyme, sIL2Rc: soluble interleukin-2 receptor, TSH: thyroid stimulating hormone, $\mathrm{FT}_{3}$ : free triiodothyronine, $\mathrm{FT}_{4}$ free thyroxine, ACTH: adrenocorticotropic hormone, DHEA-S: dehydroepiandrosterone sulfate, PRA: plasma renin activity, PAC: plasma aldosterone concentration, IGF-1: insulin-like growth factor 1, iPTH: intact parathyroid hormone

single strand DNA IgG antibodies (data not shown). The patient did not exhibit symptoms that suggested collagen diseases, which indicated that this result was not specific to the patient's condition. The patient asked to stop the nivolumab therapy, and he was subsequently discharged with hydrocortisone replacement therapy $(15 \mathrm{mg} /$ day $)$.

Follow-up MRI at three months after the discharge revealed no apparent changes in the patient's status. We also observed normalized levels of sodium, basal TSH ( $2.98 \mu \mathrm{IU} /$ $\mathrm{mL})$, PRA (3.3 ng/mL/hr), and PAC (60.9 pg/mL). However, we did not observe any other remarkable changes in the other anterior pituitary hormone levels, with very low levels of ACTH and cortisol $(<1.0 \mathrm{pg} / \mathrm{mL}$ and $1.6 \mu \mathrm{g} / \mathrm{dL}$, respectively). The patient is currently alive and has not re-started nivolumab therapy.

\section{Discussion}

In the present case, we initially used MRI and biomarkers to exclude various possible causes of pituitary dysfunction, such as pituitary or hypothalamic metastasis of the original lung cancer, a primary pituitary tumor, sarcoidosis, or malignant lymphoma. A pituitary biopsy was not performed because of the patient's advanced age and performance status. Interestingly, the increased usage of ICIs among patients with malignant disease has resulted in a growing number of reports regarding endocrine dysfunction (9). Pituitary dysfunction has been variably associated with ICI treatment for various cancers, and the different incidences may be related to the specific agent, dose, and cancer type (7). Despite the large differences in the incidences of hypopituitarism among studies, it is more frequent in cases of ipilimumab therapy 

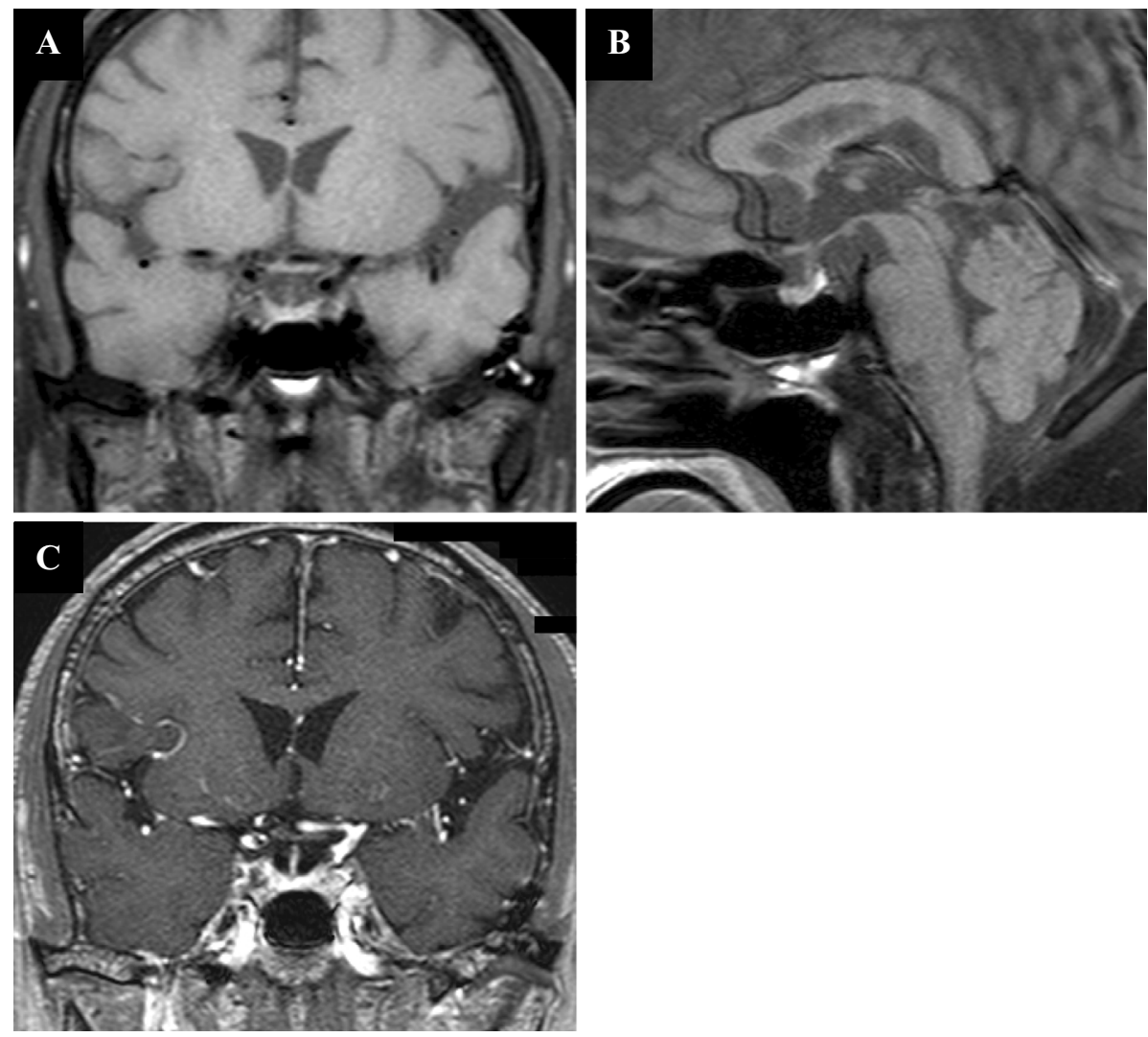

Figure 1. A: Coronal section of a T1-weighed magnetic resonance image (MRI) scan of the brain reveals no enlargement of the pituitary gland or the stalk's thickness. B: Sagittal section of a T1weighed brain MRI reveals a normal signal at the posterior lobe of the pituitary gland. C: Coronal section of a contrast-enhanced T1-weighed MRI scan of the brain reveals no abnormality in the pituitary gland.

than in those with other ICIs (7). Indeed, recent studies have suggested that $10-15 \%$ of patients who receive ipilimumab therapy may develop hypopituitarism (5), although the pathological basis of the hypopituitarism remains poorly characterized, as no pituitary histological specimens have been obtained in human cases of ICI-induced hypopituitarism (5). Furthermore, although a definitive diagnosis of hypophysitis depends on microscopic evidence, a presumptive diagnosis is generally based on newly diagnosed hypopituitarism (without an alternative etiology) and reversible radiographic pituitary enlargement $(5,10)$. Longitudinal case cohorts have revealed that 88 of 112 cases (78.6\%) with ipilimumab-induced hypopituitarism exhibited radiographic enlargement of the pituitary gland. However, considering that the enlargement can be quite mild or transient and may not be readily apparent without comparison to prior imaging findings (5), morphological changes in the pituitary gland (i.e. enlargement) can exist in the vast majority of cases. In addition, Faje et al. observed resolution of the pituitary enlargement in all of their patients (5). Considering these findings, we can infer that the pathologic basis of ipilimumabinduced hypopituitarism is hypophysitis as an endocrine IRAE.

Hypophysitis is rare among patients who are treated using other ICIs (5), and the thyroid gland is the most commonly affected endocrine organ in cases with nivolumab and pembrolizumab treatment (7). For example, only 18 hypophysitis cases $(0.9 \%)$ were identified in 10 studies that included approximately 2,000 patients receiving nivolumab, pembrolizumab, or anti-PD-L1 (5). Furthermore, nivolumab-associated all-grade pituitary dysfunction only occurred in $0.5-0.9 \%$ of reported cases $(1,6,11-16)$, and there are only 3 related case reports (17-19). Table 2 summarizes the clinical profiles of those patients and the present patient. Interestingly, the nivolumab-induced cases took much longer to diagnose than the ipilimumab-induced cases (5). Radiographic pituitary enlargement was detected in only one of four cases, suggesting that morphologic changes in the pituitary may be less severe in nivolumab-induced hypophysitis patients than that in ipilimumab-induced hypophysitis patients. A previous report described secretion dysfunction for TSH, ACTH, LH/ FSH, and PRL in $92.0 \%, 74.0 \%, 85.7 \%$, and $67.7 \%$ of ipilimumab-induced hypophysitis cases, respectively (5), whereas TSH secretion dysfunction was only observed in 2 of 4 nivolumab cases, and no cases exhibited LH/FSH secretion dysfunction (Table 2). This information, along with the radiographic characteristics, suggests that the inflammatory process in the pituitary gland is much milder in nivolumabinduced hypophysitis than in ipilimumab-induced hypophysitis. 

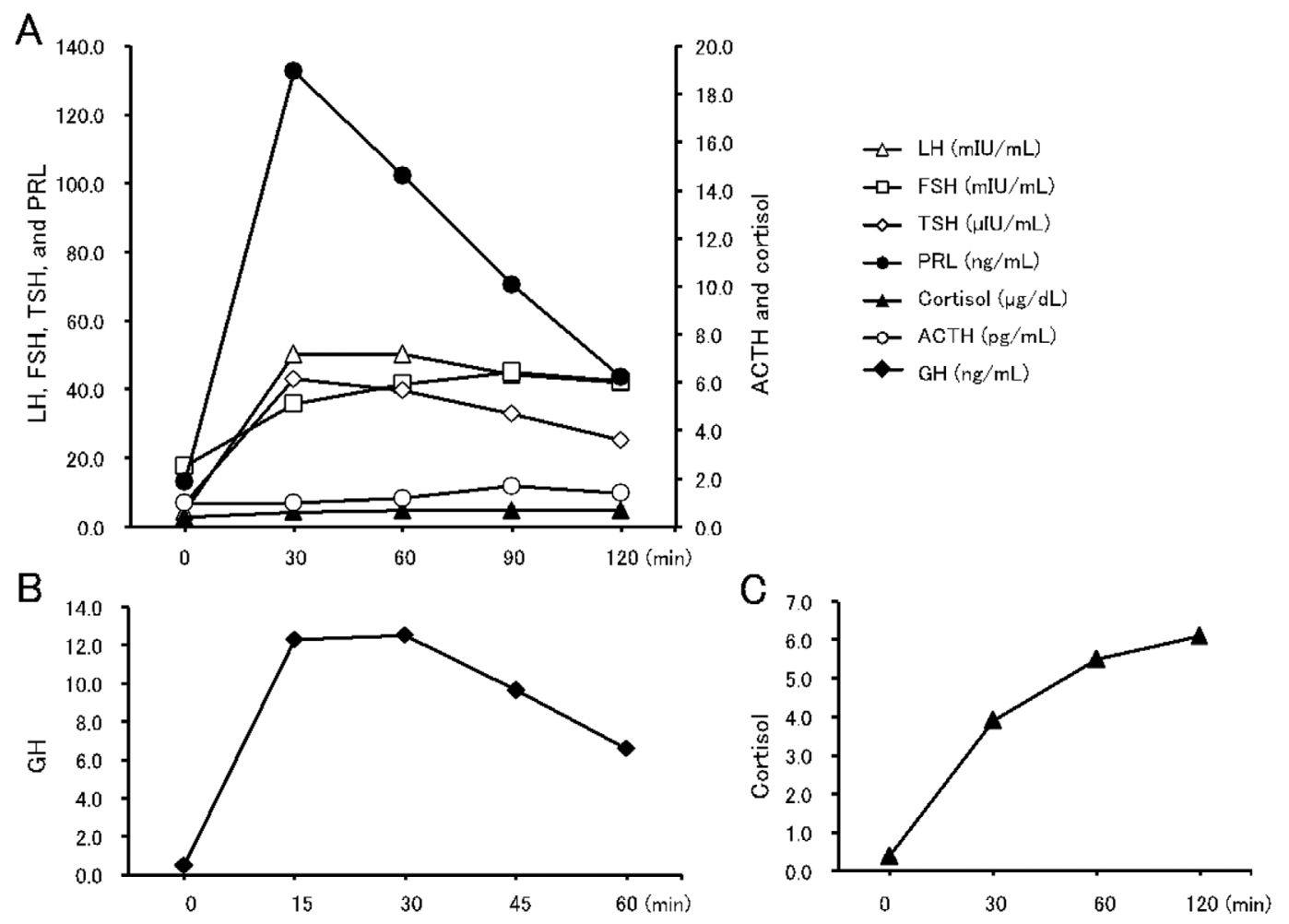

Figure 2. A: Pituitary stimulation test results (by corticotropin-releasing hormone, luteinizing hormone-releasing hormone, and thyrotropin-releasing hormone). B: Growth hormone-releasing peptide-2 test. C: Rapid adrenocorticotropic hormone test results. ACTH: adrenocorticotropic hormone, LH: luteinizing hormone, FSH: follicle-stimulating hormone, TSH: thyroid-stimulating hormone, PRL: prolactin, GH: growth hormone

Table 2. Reports Regarding Nivolumab-induced Hypopituitarism.

\begin{tabular}{lcccc}
\hline & Case 1 (17) & Case 2(18) & Case 3 (19) & Case 4 (Present case) \\
\hline Male/Female & male & male & male & male \\
Age & 55 & 68 & 50 & 75 \\
Primary disease & $\mathrm{MM}$ & $\mathrm{MM}$ & $\mathrm{MM}$ & $\mathrm{NSCLC}$ \\
Dosage (/kg) & $2 \mathrm{mg} / 3 \mathrm{w}$ & $2 \mathrm{mg} / 3 \mathrm{w}$ & $2 \mathrm{mg} / 3 \mathrm{w}$ & $3 \mathrm{mg} / 2 \mathrm{w}$ \\
Cycles of nivolumab therapy & 4 & $\mathrm{ND}$ & 6 & 12 \\
Time after nivolumab initiation & 12 weeks & 28 weeks & 16 weeks & 23 weeks \\
Radiographic pituitary enlargement & $(-)$ & $(-)$ & $(+)$ & $(-)$ \\
Hyponatremia & $(-)$ & $\mathrm{ND}$ & $(+)$ & $(+)$ \\
Hypoglycemia & $(-)$ & $\mathrm{ND}$ & $(-)$ & $(-)$ \\
Hypotension & $(+)$ & $\mathrm{ND}$ & $\mathrm{ND}$ & $(+)$ \\
Anterior pituitary dysfunction & & & & $(-)$ \\
TSH & $(-)^{*}$ & $(-)$ & $(+)$ & $(+)$ \\
ACTH & $(+)$ & $(+)$ & $(+)$ & $(-)$ \\
LH/FSH & $(-)^{*}$ & $(-)$ & $(-)$ & $(-)$ \\
GH & $(-)^{*}$ & $(-)$ & $(-)$ & $(-)$ \\
PRL & $(-)^{*}$ & $(-)$ & $(+$, elevated & $(+$, hyper-responsive) \\
Posterior pituitary dysfunction & $(-)$ & $(-)$ & $(-)$ & $(-)$ \\
\hline
\end{tabular}

MM: malignant melanoma, NSCLC: non-small-cell lung carcinoma, TSH: thyroid stimulating hormone, ACTH: adrenocorticotropic hormone, LH: luteinizing hormone, FSH: follicle-stimulating hormone, GH: growth hormone, PRL: prolactin, ND: not described, ${ }^{*}$ diagnosed by basal hormone levels, ${ }^{* *}$ normal response in the stimulation test

The mechanisms responsible for ICI-induced hypophysitis and the pathogenetic differences between ipilimumab and other ICIs are unclear. However, the autoimmune pathogenesis of this adverse effect has recently been supported by the identification of anti-thyrotroph, anti-corticotroph, and antigonadotroph antibodies in the sera of patients with ipilimumab-induced hypophysitis (20). Furthermore, a mouse model of hypophysitis induced using a CTLA-4 
blocking antibody revealed mononuclear cell infiltration in the pituitary gland but not in other organs, which suggests that this mechanism may be specific for the pituitary gland (20). A pathogenic role of the classic complement pathway has also been suggested, as CTLA-4 expression with $\mathrm{C} 3, \mathrm{C} 3 \mathrm{~d}$, and $\mathrm{C} 4 \mathrm{~d}$ deposition was observed in mouse and human pituitary cells after exposure to anti-CTLA-4 antibodies (21). Another possible mechanism for ICI-induced hypophysitis involves the activation of antibody-dependent cell-mediated cytotoxicity (ADCC) $(21,22)$, which may explain the different incidences of hypophysitis in cases with ipilimumab or nivolumab/pembrolizumab treatment $(5,7)$. This is because ipilimumab is an IgG1 antibody that can activate both the classical complement pathway and ADCC, whereas nivolumab and pembrolizumab are IgG4 antibodies that cannot activate the classical complement pathway and are less effective in ADCC (23). The activity sites may also be relevant, as nivolumab and pembrolizumab have more peripheral activity sites than ipilimumab, and CTLA-4 is involved in early T-lymphocyte tolerance while the PD-1 pathway plays less important roles in regulating peripheral Tlymphocyte tolerance and inflammation (24). Therefore, it is reasonable that patients might better tolerate PD-1 pathwayblocking agents than CTLA-4 blockade agents (24), a point that also agrees with nivolumab and pembrolizumab being associated with a lower rate of IRAEs (vs. ipilimumab) (25).

The risk factors for ICI-induced hypophysitis also remain unclear, as idiopathic autoimmune hypophysitis is relatively common among women (26), while male sex and older age may be risk factors for the development of ipilimumabinduced hypophysitis $(9,27,28)$. Concurrent treatment using non-ICI agents does not appear to increase this risk (5), and the incidence of ipilimumab-induced hypophysitis is not significantly elevated among patients who receive combination therapy using nivolumab $(29,30)$. It is also unclear whether patients with ICI-induced hypophysitis have an increased risk of other IRAEs, and additional treatment cycles are not routinely contraindicated in patients with ICI-induced hypophysitis (5).

Treatment for ICI-induced hypophysitis includes the initiation of glucocorticoid and thyroid hormone replacement early after the diagnosis of hypophysitis (5). Androgen replacement can also be considered later if hypogonadism persists, although treatment using growth hormone is contraindicated in patients with ICI-induced hypophysitis because of the original malignant disease (5). The initial treatment for ICI-induced hypophysitis includes high-dose glucocorticoid therapy (approximately $1 \mathrm{mg} / \mathrm{kg}$ body weight prednisolone or equivalent) (9). However, the overall failure and recurrence rates are elevated among patients with idiopathic hypophysitis who receive high-dose glucocorticoid therapy, although the initial response has been favorable (31). Similarly, a recent study of ipilimumab-induced hypophysitis revealed that high-dose glucocorticoid therapy did not appear to improve the outcomes (e.g. pituitary function recovery or the overall survival) (27). Interestingly, the antitumor effects of ipilimumab do not seem not to be affected by early treatment using systemic high-dose glucocorticoids $(32,33)$, although prolonged glucocorticoid treatment for IRAEs also did not appear to affect the survival among patients who received ipilimumab for melanoma (34).

Isolated ACTH deficiency has been reported to be associated with several hormonal abnormalities mainly caused by hypocortisolemia, such as high levels of TSH (35) and suppression of the renin-angiotensin system due to inappropriate secretion of vasopressin (36). Although we observed elevated TSH and suppressed plasma renin activity in the present case, both were normalized after the initiation of hydrocortisone therapy. We also observed slightly elevated PAC at his admission; however, its clinical significance was unclear because it was also normalized by hydrocortisone therapy.

In conclusion, we reported a case of isolated-ACTH deficiency that was induced by nivolumab treatment for metastatic lung cancer. Therefore, awareness of possible endocrine complications (especially hypophysitis and subsequently adrenal insufficiency) is essential for oncologists and endocrinologists based on the increasing use of ICIs in patients with malignant diseases. Patients who receive nivolumab therapy also should be informed of this rare but lifethreatening adverse effect in advance. Furthermore, regular follow-up testing of the endocrine function should be mandatory for patients treated using ICIs.

\section{The authors state that they have no Conflict of Interest (COI).}

\section{References}

1. Naidoo J, Page DB, Li BT, et al. Toxicities of the anti-PD-1 and anti-PD-L1 immune checkpoint antibodies. Ann Oncol 26: 23752391, 2015.

2. Nishimura H, Honjo T. PD-1: an inhibitory immunoreceptor involved in peripheral tolerance. Trends Immunol 22: 265-268, 2001.

3. Sharma P, Wagner K, Wolchok JD, Allison JP. Novel cancer immunotherapy agents with survival benefit: recent successes and next steps. Nat Rev Cancer 11: 805-812, 2011.

4. ClinicalTrials.gov. Available from: https://clinicaltrials.gov

5. Faje A. Immunotherapy and hypophysitis: clinical presentation, treatment, and biologic insights. Pituitary 19: 82-92, 2016.

6. Topalian SL, Hodi FS, Brahmer JR, et al. Safety, activity, and immune correlates of anti-PD-1 antibody in cancer. N Engl J Med 366: 2443-2454, 2012.

7. Torino F, Corsello SM, Salvatori R. Endocrinological side-effects of immune checkpoint inhibitors. Curr Opin Oncol 28: 278-287, 2016.

8. Voskens CJ, Goldinger SM, Loquai C, et al. The price of tumor control: an analysis of rare side effects of anti-CTLA-4 therapy in metastatic melanoma from the ipilimumab network. PLoS One 8: e53745, 2013.

9. Corsello SM, Barnabei A, Marchetti P, De Vecchis L, Salvatori R, Torino F. Endocrine side effects induced by immune checkpoint inhibitors. J Clin Endocrinol Metab 98: 1361-1375, 2013.

10. Glezer A, Bronstein MD. Pituitary autoimmune disease: nuances in clinical presentation. Endocrine 42: 74-79, 2012.

11. Brahmer J, Reckamp KL, Baas $P$, et al. Nivolumab versus docetaxel in advanced squamous-cell non-small-cell lung cancer. $\mathrm{N}$ 
Engl J Med 373: 123-135, 2015.

12. Gettinger SN, Horn L, Gandhi L, et al. Overall survival and longterm safety of nivolumab (anti-programmed death 1 antibody, BMS-936558, ONO-4538) in patients with previously treated advanced non-small-cell lung cancer. J Clin Oncol 33: 2004-2012, 2015.

13. Motzer RJ, Rini BI, McDermott DF, et al. Nivolumab for metastatic renal cell carcinoma: results of a randomized phase II trial. J Clin Oncol 33: 1430-1437, 2015.

14. Rizvi NA, Mazieres J, Planchard D, et al. Activity and safety of nivolumab, an anti-PD-1 immune checkpoint inhibitor, for patients with advanced, refractory squamous non-small-cell lung cancer (CheckMate 063): a phase 2, single-arm trial. Lancet Oncol 16: 257-265, 2015.

15. Weber JS, D'Angelo SP, Minor D, et al. Nivolumab versus chemotherapy in patients with advanced melanoma who progressed after anti-CTLA-4 treatment (CheckMate 037): a randomised, controlled, open-label, phase 3 trial. Lancet Oncol 16: 375-384, 2015.

16. Robert C, Long GV, Brady B, et al. Nivolumab in previously untreated melanoma without BRAF mutation. $\mathrm{N}$ Engl J Med 372: 320-330, 2015.

17. Ishikawa M, Oashi K. Case of hypophysitis caused by nivolumab. J Dermatol 44: 109-110, 2017.

18. Fujimura T, Kambayashi Y, Furudate $S$, et al. Isolated adrenocorticotropic hormone deficiency possibly caused by nivolumab in a metastatic melanoma patient. J Dermatol 44: e13-e14, 2017.

19. Okano Y, Satoh T, Horiguchi K, et al. Nivolumab-induced hypophysitis in a patient with advanced malignant melanoma. Endocr J 63: 905-912, 2016.

20. Iwama S, De Remigis A, Callahan MK, Slovin SF, Wolchok JD, Caturegli P. Pituitary expression of CTLA-4 mediates hypophysitis secondary to administration of CTLA-4 blocking antibody. Sci Transl Med 6: 230ra245, 2014.

21. Laurent S, Queirolo P, Boero S, et al. The engagement of CTLA-4 on primary melanoma cell lines induces antibody-dependent cellular cytotoxicity and TNF-alpha production. J Transl Med 11: 108, 2013.

22. Romano E, Kusio-Kobialka M, Foukas PG, et al. Ipilimumabdependent cell-mediated cytotoxicity of regulatory $\mathrm{T}$ cells ex vivo by nonclassical monocytes in melanoma patients. Proc Natl Acad Sci U S A 112: 6140-6145, 2015.

23. Vidarsson G, Dekkers G, Rispens T. IgG subclasses and allotypes: from structure to effector functions. Front Immunol 5: 520, 2014.

24. Mahoney KM, Freeman GJ, McDermott DF. The next immunecheckpoint inhibitors: PD-1/PD-L1 blockade in melanoma. Clin Ther 37: 764-782, 2015.

25. Michot JM, Bigenwald $C$, Champiat $S$, et al. Immune-related adverse events with immune checkpoint blockade: a comprehensive review. Eur J Cancer 54: 139-148, 2016.

26. Caturegli P, Newschaffer C, Olivi A, Pomper MG, Burger PC, Rose NR. Autoimmune hypophysitis. Endocr Rev 26: 599-614, 2005.

27. Min L, Hodi FS, Giobbie-Hurder A, et al. Systemic high-dose corticosteroid treatment does not improve the outcome of ipilimumabrelated hypophysitis: a retrospective cohort study. Clin Cancer Res 21: 749-755, 2015.

28. Faje AT, Sullivan R, Lawrence D, et al. Ipilimumab-induced hypophysitis: a detailed longitudinal analysis in a large cohort of patients with metastatic melanoma. J Clin Endocrinol Metab 99: 4078-4085, 2014.

29. Wolchok JD, Kluger $\mathrm{H}$, Callahan $\mathrm{MK}$, et al. Nivolumab plus ipilimumab in advanced melanoma. N Engl J Med 369: 122-133, 2013.

30. Postow MA, Chesney J, Pavlick AC, et al. Nivolumab and ipilimumab versus ipilimumab in untreated melanoma. $\mathrm{N}$ Engl $\mathrm{J}$ Med 372: 2006-2017, 2015.

31. Honegger J, Buchfelder M, Schlaffer S, et al. Treatment of primary hypophysitis in germany. J Clin Endocrinol Metab 100: 3460-3469, 2015.

32. Horvat TZ, Adel NG, Dang TO, et al. Immune-related adverse events, need for systemic immunosuppression, and effects on survival and time to treatment failure in patients with melanoma treated with ipilimumab at Memorial Sloan Kettering Cancer Center. J Clin Oncol 33: 3193-3198, 2015.

33. Boasberg P, Hamid O, O'Day S. Ipilimumab: unleashing the power of the immune system through CTLA-4 blockade. Semin Oncol 37: 440-449, 2010.

34. Johnson DB, Friedman DL, Berry E, et al. Survivorship in immune therapy: assessing chronic immune toxicities, health outcomes, and functional status among long-term ipilimumab survivors at a single referral center. Cancer Immunol Res 3: 464-469, 2015.

35. Hannon MJ, O'Halloran DJ. Isolated acquired ACTH deficiency and primary hypothyroidism: a short series and review. Pituitary 14: 358-361, 2011.

36. Saito T, Tojo K, Yamamoto H, Hosoya T, Tajima N. Isolated adrenocorticotropin deficiency presenting with impaired reninangiotensin-aldosterone system and suppressed parathyroid hormone-vitamin D axis. Intern Med 41: 561-565, 2002.

The Internal Medicine is an Open Access article distributed under the Creative Commons Attribution-NonCommercial-NoDerivatives 4.0 International License. To view the details of this license, please visit (https://creativecommons.org/licenses/ by-nc-nd/4.0/).

(C) 2017 The Japanese Society of Internal Medicine Intern Med 56: 2463-2469, 2017 